\title{
Testing Absolute $v s$ Relative Simultaneity with the Spin-orbit Interaction and the Sagnac Effect
}

\author{
Gianfranco Spavieri ${ }^{1}$, Miguel Rodriguez ${ }^{2} \&$ Arturo Sanchez ${ }^{3}$
}

\author{
${ }^{1}$ Centro de Fłsica Fundamental, Facultad de Ciencias, Universidad de Los Andes, Mrida, 5101-Venezuela \\ 2 Ecological World For Life GmbH, D-15537 Erkner, Germany \\ ${ }^{2}$ Istituto Nazionale di Fisica Nucleare (INFN), Università di Udine, International Centre for Theoretical Physics, Trieste, \\ Italy
}

Correspondence: Gianfranco Spavieri, Centro de Fłsica Fundamental, Facultad de Ciencias, Universidad de Los Andes, Mérida, 5101-Venezuela. E-mail: gspavieri@gmail.com

\author{
Received: April 9, $2019 \quad$ Accepted: May 30, $2019 \quad$ Online Published: July 19, 2019 \\ doi:10.5539/apr.v11n4p59 URL: https://doi.org/10.5539/apr.v11n4p59
}

\begin{abstract}
All the experiments supporting special relativity (SR) formulated with Einstein synchronization support as well SR with absolute synchronization, if the corresponding coordinate transformations foresee time dilation and length contraction. We first test absolute $v s$ relative simultaneity with a non-relativistic model of the spin-orbit interaction by taking into account either the effect of the electron hidden momentum or the relativistic effect of the Thomas precession, based on non-conservation of simultaneity. As second test, we consider a thought experiment equivalent to the Sagnac effect, where a clock measures the time taken by a counter-propagating light signal to perform a round trip on a closed path. While these experiments are coherently described with absolute simultaneity, the result of our tests points out inconsistencies in the case of relative simultaneity, thus favoring the formulation of SR with absolute synchronization, while advocating that further research and tests on simultaneity are needed for the comprehension of relativistic theories.
\end{abstract}

Keywords: Einstein synchronization, hidden momentum, spin-orbit interaction, one-way speed of light, Sagnac effect

\section{Introduction}

According to the conventionalist thesis, special relativity (SR) theory can be formulated with either Einstein or absolute clock synchronization (Mansouri \& Sexl, 1977; de Abreu \& Guerra, 2005; Bell, 1988; Anderson, Vetharaniam \& Stedman, 1998). Until recently, the two synchronizations were considered to be physically equivalent, because all the experiments supporting SR with Einstein synchronization (standard SR) support as well SR with absolute synchronization. In effect, what is ruled out by experiment is not absolute simultaneity, but the Galileo transformations, because they do not foresee time dilation and length contraction. Yet, a recent work by Spavieri, Rodriguez and Sanchez (2018), shows that the two synchronizations can be discriminated on the basis of kinematics. Thus, in this scenario, it appears crucial to verify if there are experiments that support, or rule out, either absolute or relative simultaneity. We know of no dedicated experiments capable of testing directly absolute $v s$ relative simultaneity. Since the relativistic effects of time dilation and length contraction are present in both the Lorentz transformations (LT) and the transformations adopting absolute synchronization (AT), (Tangherlini, 1961), (Mansouri \& Sexl, 1977), (Spavieri, Rodriguez \& Sanchez, 2018), both LT and AT interpret the existing experiments. Thus, the effects due to the difference between absolute and relative simultaneity are probably quite subtle and not easily detectable. For example, if the Dirac equation is assumed to be valid in the frame where an atom is at rest, the predicted atomic energy levels are the same, independently of the synchronization adopted by SR. Modifications to electrodynamics, if formulated with absolute synchronization, have been considered (Spavieri, 2012), but no discriminating experiments have been performed yet. Therefore, at the moment, we are bound to restrict ourselves to re-examine the experiments supporting SR and select those suitable for discriminating absolute from relative simultaneity. One of these experiments, referring to the measurement of the spin-orbit energy splitting in atoms, appears to be worth considering because it can be indirectly related either to the hidden momentum of the electron (Spavieri \& Mansuripur, 2015), (Spavieri, 2016), or to the relativistic interpretation of the spin precession by Thomas (Thomas, 1926), based on non-conservation of simultaneity. Another experiment is the Sagnac effect (Sagnac, 1913), whose interpretation, directly in terms of absolute or relative simultaneity, is quite controversial due to the existence of the Selleri paradox (Selleri, 1997). The preliminary conclusion that can be drawn from our analysis is that these physical phenomena are coherently described, without inconsistencies, by absolute simultaneity only. Nevertheless, what these discussions and the 
findings of Ref.(Spavieri, Rodriguez \& Sanchez, 2018) ultimately indicate, is that the scenario of relativistic theories may be changing and broadening. SR is routinely used employing the Lorentz transformations based on relative simultaneity. If SR with absolute simultaneity is used instead, by comparison new physical insights will emerge (Spavieri, Rodriguez \& Sanchez, 2018), improving our knowledge of relativistic theories and likely leading to innovative tests discriminating relative from absolute simultaneity.

\section{Spin-orbit Interaction Energy, the Thomas Precession, and the Hidden Momentum}

Before the development of relativistic quantum mechanics, in 1926 Thomas (Thomas, 1926) introduced his famous $1 / 2$ factor (the "Thomas half') to the electromagnetic interaction energy $U=-\mu \cdot \mathbf{B}^{\prime}$ of the spin-orbit coupling, where $\mu$ is the magnetic moment of the electron and $\mathbf{B}^{\prime} \cong-(\mathbf{v} / c) \times \mathbf{E}$ is the effective magnetic field (obtained by a relativistic transformation of the field $\mathbf{E}$ of the nucleus to the rest-frame of the electron). The reason for doing this was that the value $U$ given by classical electrodynamics leads to a spin-orbit energy splitting, $\Delta \mathcal{E}_{s o}$, that is twice as large as that experimentally observed (Jackson, 1999). Thus, Thomas formulated a precession mechanism (the 'Thomas precession') based on successive relativistic transformations to the frame co-moving with the electron in its circular orbit, by which the overall interaction energy reduces to, $\Delta \mathcal{E}_{s o}=(1 / 2) U$, in agreement with observation. Considered for already almost a century as the dominant approach to spin-orbit interaction, Thomas' mechanism has been adopted in treatises, papers, and most textbooks of classical electrodynamics, modern physics, and quantum mechanics. Moreover, under appropriate assumptions (Jackson, 1999), Thomas' precession can be included (Jackson, 1999) in the equation of motion of spin, as is the case for the BMT equation (Bargmann, Michel \& Telegdi, 1959), used in elementary particle physics. Therefore, in order to foresee the correct spin-orbit energy splitting, the non-relativistic equations of atomic physics, describing the electron orbiting the nucleus, have to introduce the Thomas precession, which is a relativistic effect due to nonconservation of simultaneity derived in the frame co-moving with the electron.

\subsection{The Hidden Momentum}

However, the evolution of classical electrodynamics exposed an important aspect that can be related to the interpretation of spin-orbit interaction and refers to the correct force on a particle possessing a magnetic dipole-moment $\mu$. Near the end of the eighties, the correct force acting on a moving dipole has been found (Aharonov, Pearle \& Vaidman, 1988), incorporating the force contribution due to the hidden momentum

$$
\mathbf{P}_{h}=c^{-1} \mu \times \mathbf{E},
$$

related to the interaction electromagnetic momentum $\mathbf{P}_{e m}=(1 / 4 \pi c) \int(\mathbf{E} \times \mathbf{B}) d \tau=-\mathbf{P}_{h}$. The hidden momentum $\mathbf{P}_{h}=$ $-\mathbf{P}_{e m}$ of the magnetic dipole is formed in the interaction of the electric field $\mathbf{E}$ of a charge $q$ with the field $\mathbf{B}$ of a magnetic dipole $\mu$. By taking into account the electric field $\mathbf{E}$ of the nucleus and using the correct expression of the force on the magnetic moment of the electron, Spavieri and Mansuripur (Spavieri \& Mansuripur, 2015), and Spavieri (Spavieri, 2016) developed a semi-classical model of the spin-orbit interaction that, when evaluated in the frame of the nucleus, leads to the expected value for the spin-orbit coupling energy. In effect, although the electromagnetic interaction energy is $U$, in a pure spin flip the total energy changes only by $(1 / 2) U$, the factor $1 / 2$ emerging naturally without the need of the additional relativistic Thomas' mechanism derived in the frame co-moving with the electron. It is worth remarking that the role of $\mathbf{P}_{e m}$ and $\mathbf{P}_{h}$ is central for comprehending aspects of quantum non-locality in the context of the effects of the Aharonov-Bohm type (Spavieri, 1998). In these non-local effects, where the $q-\mu$ (or $\mathbf{E}-\mathbf{B}$ ) interaction takes place, the observable phase shift is related to $\mathbf{P}_{e m}$ (or $\mathbf{P}_{h}$ ) and to the properties of the related angular momentum of electromagnetic fields. These features have led to the formulation of new effects of the Aharonov-Bohm type, such as a quantum effect for electric dipoles (Spavieri, 1998). Moreover, the hidden momentum $\mathbf{P}_{h}$ has been decisive in finding the correct force on the magnetic dipole (Aharonov, Pearle \& Vaidman, 1988), solving the Shokley-James paradox (Shockley \& James, 1967), (Aharonov, Pearle \& Vaidman, 1988), and corroborating the nonlocality of the Aharonov-Casher quantum effect (Aharonov, Pearle \& Vaidman, 1988).

\subsection{Concluding Remarks}

At this point, if the effect of the hidden momentum is taken into account, the additional contribution due to the Thomas precession in the spin-orbit interaction gives a wrong result. Several arguments can be advanced in favor of (or against) the Thomas precession mechanism or the hidden momentum concept. We consider convenient to discuss in the Appendix some of the objections that can be raised against the derivation of the Thomas precession that appear in textbooks. About the factor $1 / 2$ to be derived within semi-classical models of the atom by means of Thomas' approach, we mention here the objection made by Kholmetskii, Missevitch, and Yarman (2010). According to these authors, there is a 'logical inconsistency' in the semi-classical models of spin-orbit splitting, relying on a non-relativistic equation of motion while including the relativistic phenomenon of Thomas' precession. This inconsistency is avoided with the approach that introduces the hidden momentum and the correct force on the electron magnetic dipole. Then, the correct factor $1 / 2$ is 
derived in the rest-frame of the nucleus, without having to rely on the relativistic Thomas' precession mechanism or other relativistic quantum effects. The above considerations leads to the conclusion that the spin-orbit interaction possesses a non-relativistic semi-classical origin, favoring the hidden momentum approach over the Thomas precession mechanism.

In view of the fact that tests of $\mathbf{P}_{h}, \mathbf{P}_{e m}$, and the angular momentum $\boldsymbol{\Gamma}_{\text {mech }}$, are feasible (Spavieri, G., 2016), (Spavieri, G., 2012), and given the renewed interest over the hidden momentum concept during the past few years, it is worth mentioning works of physicists either invoking the idea of hidden momentum (Khorrami \& Saldanha, 2013) or arguing against the need for hidden momentum (Kemp, 2015). The lack of experiments testing $\mathbf{P}_{h}, \mathbf{P}_{e m}, \boldsymbol{\Gamma}_{m e c h}$, and other fundamental aspects of electromagnetism, reflects the existence of different untested interpretations of electrodynamics, such as MaxwellLorentz's theory and the Einstein-Laub viewpoint. If experiments confirm the validity of the hidden momentum concept or the Einstein-Laub viewpoint, it would further support the interpretation of the spin-orbit mechanism in terms of absolute simultaneity rather than in terms of the relativistic Thomas precession based on the relativity of simultaneity.

\section{The Sagnac Effect and the One-way Speed of Light}

Among optical tests and other basic tests of SR, the Sagnac effect is relevant because its outcome is of first order in $v / c$. In the Sagnac effect, a disk of radius $R$ is rotating at constant angular velocity $\omega$ and an observer with a clock $\mathrm{O}^{\mathrm{T}}$ at a point on the circumference is co-moving with it at the speed $v=\omega R$. As indicated in the upper part of Fig. 1, this observer sends two counter-propagating light signals around the disk circumference and measures the relative propagation time delay $\Delta t$. In the laboratory frame $S$, where the one-way speed is $c$ and the center of the disk is stationary, the description of the Sagnac effect, and corresponding time span variation $\Delta t=t_{-}-t_{+}$for counter-propagating signals, is the same for Newtonian physics and relativistic theories. To first order in $v / c$ and with $2 \pi R=2 L$, the propagation time $t_{+}$and $t_{-}$of the signals are,

$$
t_{+} \simeq \frac{2 L}{c+v} ; t_{-} \simeq \frac{2 L}{c-v} ; \Delta t \simeq \frac{4 L v}{c^{2}} .
$$

Then, the average speeds of light in the counter- and co-moving senses are

$$
c_{ \pm}=\frac{2 L}{t_{ \pm}} \simeq c \pm v .
$$

a)

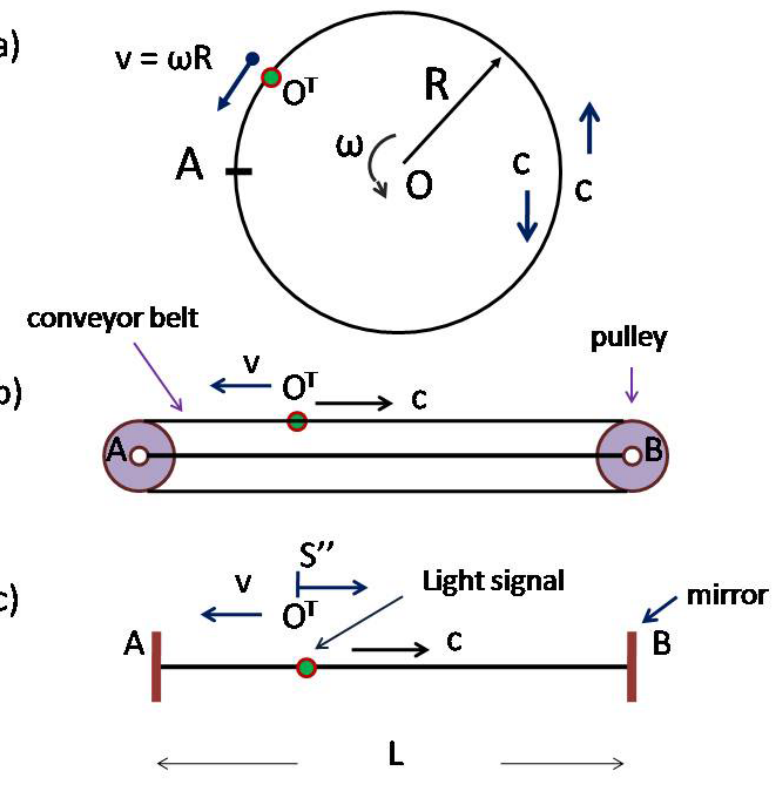

Figure 1:a): The Sagnac effect with interferometer (or clock $\mathrm{O}^{\mathrm{T}}$ ) on a rotating disk and light signals counter-propagating on the circumference. $b$ ): Experimental set-up resembling a conveyor belt with clock $\mathrm{O}^{\mathrm{T}}$ co-moving with the belt sliding on the pulleys at $\mathrm{A}$ and $\mathrm{B}$. The light signal is counter-moving along the belt section of length $\mathrm{AB}=\mathrm{L}$. $c$ ): The upper part of the sliding belt is shown as seen in the rest frame $S$ of the arm $\mathrm{AB}$. Clock $\mathrm{O}^{\mathrm{T}}$ moves with velocity $-v$ toward point $\mathrm{A}$ while the light signal emitted by $\mathrm{O}^{\mathrm{T}}$ travels toward point $\mathrm{B}$ where is reflected by a mirror. When $\mathrm{O}^{\mathrm{T}}$ reaches $\mathrm{A}$, it changes direction and is co-moving with the lower part of the belt. 
Originally, the Sagnac effect was thought to disprove special relativity (Sagnac, 1913). Indeed, the velocity difference of the average velocities in (3), leads to a paradox according to Selleri (1997), who shows that, when the radius of the rotating disk is increased to $R \rightarrow \infty$ keeping constant $\omega R=v$, the local speed of light in an inertial frame would be $c+v$ or $c-v$ depending on the direction of propagation, in contrast with the second postulate of SR.

In rebutting Selleri's claim, Kassner (2012) attempts to refute the paradox by first interpreting the Sagnac effect with a Minkowsky analysis, but settles by recognizing that "Einstein synchronization fails when performed along a path around a full circle", i.e., a closed path. The same failure has also been pointed out by Weber (1997) and earlier by Anandan (1981). Then, in order to account for the discontinuity related to the speeds $c+v$ and $c-v$, Kassner introduces the unusual concept of a "time gap" and states that "the speed of light is $c$ everywhere except at the point on the circle where we put the time gap." Kassner proposal of an adjustable time gap has been objected to by Gift (2015), arguing that it is untenable because based on an "unphysical time discontinuity". A second interesting argument presented by Kassner (2012) to solve Selleri's paradox (1997), consists of replacing Einstein synchronization with absolute synchronization. The same argument has been used by Mansouri and Sexl (1977) to solve the clock (or "twin") paradox. Then, in line with the conventionalist thesis, wherein Einstein and absolute synchronizations are physically equivalent, Kassner argues that the difference in the speeds $c+v$ and $c-v$, is to be "interpreted in terms of the arbitrariness of synchronization" and, thus, does not invalidate the second postulate of SR. Yet, in general, Einstein and absolute synchronizations are not physically equivalent (Spavieri, Rodriguez \& Sanchez, 2018). Only one of the two is correct! Thus, Kassner's argument based on the arbitrariness of synchronization is untenable.

\section{An Experimental Set-up Suitable for Verifying "Ground" Local Speeds}

"Linearized" versions of the Sagnac effect have been considered in Refs. (Wang, Zhengb, Yaob \& Langley, 2003) and (Spavieri \& et al., 2019), where light propagates in a "conveyor belt" system (or moving fiber) equivalent to the circular rotating disk system. For convenience of the reader, we present here the details of a possible version of the "linear" Sagnac effect suitable for testing absolute $v s$ relative simultaneity. Thus, in Fig. 1 we consider the arm AB of length $L$ with two mirrors at the end points $\mathrm{A}$ and $\mathrm{B}$. A light signal is emitted from a clock, denoted by $\mathrm{O}^{\mathrm{T}}$, which measures the time $T$ taken by the signal to cover the belt "ground" path of length $2 L$. As shown in Figs. 1 and 2, we consider the counter-moving signal (emitted by clock $\mathrm{O}^{\mathrm{T}}$ ) traveling initially along the conveyor belt upper part from clock $\mathrm{O}^{\mathrm{T}}$ toward the mirror at $\mathrm{B}$, from where it is reflected back to $\mathrm{O}^{\mathrm{T}}$ propagating in the belt lower part. As indicate in Fig. $1 \mathrm{c}$ ), where we describe the light signal propagation in the upper part of the belt, relatively to the arm $\mathrm{AB}$ (frame $S$ ), clock $\mathrm{O}^{\mathrm{T}} \equiv \mathrm{O}^{\prime \prime}$ (stationary in frame $S^{\prime \prime}$ ) is first moving with velocity $-v$ from $\mathrm{O}$ to A. Then, after reaching point A (Fig. 2a)), $\mathrm{O}^{\mathrm{T}}$ changes direction and is co-moving with the belt lower part at rest in frame $S^{\prime}$ (Fig. 2 b)), which is moving with velocity $v$ relatively to $S$.

Since the observable (2) is of first order in $v / c$, we may neglect higher order terms in the Lorentz transformations $x^{\prime}=$ $\gamma_{w}\left(x^{\prime \prime}-w t^{\prime \prime}\right)$ from $S^{\prime \prime}$ to $S^{\prime}$ and, assuming $\gamma_{w} \simeq 1$, for simplicity, we use the transformations,

$$
\begin{gathered}
x^{\prime}=x^{\prime \prime}-w t^{\prime \prime} \\
t^{\prime}=t^{\prime \prime}-w x^{\prime \prime} / c^{2} \quad(\mathrm{LT}) ; \quad t^{\prime}=t \quad \text { (AT), }
\end{gathered}
$$

where in (4) and (5), $w=2 v$ to first order in $v / c$. For the time transformation, we employ the first order approximation $t^{\prime}=t^{\prime \prime}-w x^{\prime \prime} / c^{2}$ for the LT and $t^{\prime}=t$ for Newtonian physics and the AT. Clock $\mathrm{O}^{\mathrm{T}}$ measures the round trip time of flight $T$ of the signal from $\mathrm{O}^{\mathrm{T}}$ to $\mathrm{B}$ and from $\mathrm{B}$ back to $\mathrm{O}^{\mathrm{T}}$.

For our thought experiment, which may be related to the Sagnac effect, we require a single light signal only, counterpropagating relatively to $\mathrm{O}^{\mathrm{T}}$ in this case. It is important to stress that we wish to test absolute $v s$ relative simultaneity and the validity of Einstein's second postulate. If we choose to describe our thought experiment from a single frame (for example, the laboratory frame $S$ where the conveyor belt arm AB is stationary), we may assume that the local speed is $c$ in $S$, but then we are unable to check the consistency of the different synchronizations in estimating the value of the local speed (which depends on the synchronization) in the different moving sections (and co-moving frames) of the belt. Instead, for our purpose, we need to check the value of the one-way speed of light along each section of the ground path $2 L$, co-moving with either frame $S^{\prime \prime}$ or $S^{\prime}$. Thus, we need first to analyze the time measured by clock $\mathrm{O}^{\mathrm{T}}$, when $\mathrm{O}^{\mathrm{T}}$ is stationary in frame $S^{\prime \prime}$, and the corresponding value of the local speed while the signal travels in the upper section of the belt co-moving with $S^{\prime \prime}$. Then, separately and after clock $\mathrm{O}^{\mathrm{T}}$ changes direction at A and is co-moving with frame $S^{\prime}$, we need to perform the same analysis when the signal travels in the lower section of the belt co-moving with $S^{\prime}$, as shown below. 


\subsection{Propagation Time Measured by $O^{T}$ While Stationary in Frame $S^{\prime \prime}$}

Consider the clock $\mathrm{O}^{\mathrm{T}}$ co-moving with the upper part of the belt and the inertial frame $S^{\prime \prime}$, as shown in Fig. $1 \mathrm{~b}$ ). At the time when the signal is emitted by $\mathrm{O}^{\mathrm{T}}$, the initial conditions in frame $S^{\prime \prime}$, are: $\mathrm{AB}=L, \mathrm{AO}^{\prime \prime}=\mathrm{AO}^{\mathrm{T}}=v L / c$ and $\mathrm{O}^{\mathrm{T}} \mathrm{B}=$ $\mathrm{O}^{\prime \prime} \mathrm{B}=L(1-v / c)$. The coherent form for relating the readings of clock $\mathrm{O}^{\mathrm{T}}$ to the local speed $C$ in the frame where $\mathrm{O}^{\mathrm{T}}$ is at rest is,

$$
\Delta T=\Delta L / C,
$$

where $\Delta L$ is the "ground" distance covered by the signal in the time interval $\Delta T$ measured by $\mathrm{O}^{\mathrm{T}}$ and the synchronized clocks of the frame where $\mathrm{O}^{\mathrm{T}}$ is at rest. Of course, $\Delta L$ must be measured with meter sticks at rest in frame where $\mathrm{O}^{\mathrm{T}}$ is stationary. If we assume space isotropy and the one-way speed of light to be $c$ in frame $S^{\prime \prime}$ where $\mathrm{O}^{\mathrm{T}}$ is stationary, which is then also the preferred frame, the local speed is $C=c$. Since the signal is emitted from the position of $\mathrm{O}^{\mathrm{T}}$, the equation of motion of the light signal in $S^{\prime \prime}$ may be written as: $c t^{\prime \prime}=L(1-v / c)+v t^{\prime \prime}$, so that the signal reaches B in the time interval,

$$
T_{A B}=\Delta L / C=t_{A B}^{\prime \prime}=L / c,
$$

displayed by $\mathrm{O}^{\mathrm{T}} \equiv \mathrm{O}^{\prime \prime}$. Moreover, since the equation of motion of point $\mathrm{A}$ is, $-(v / c) L+v t^{\prime \prime}=0$, point $\mathrm{A}$ reaches clock $\mathrm{O}^{\mathrm{T}} \equiv \mathrm{O}^{\prime \prime}$ at the same time $T_{O^{\mathrm{T}} A}=T_{A B}=t_{A B}^{\prime \prime}=L / c$, the two events "clock $\mathrm{O}^{\mathrm{T}}$ at $\mathrm{A}$ " and "light signal at B" being simultaneous in $S^{\prime \prime}$.

\subsection{Propagation Time Measured by $O^{T}$ While Stationary in Frame $S^{\prime}$, If the Return Path Has Length $L^{\prime}=L$}

After reaching point $\mathrm{A}$, clock $\mathrm{O}^{\mathrm{T}}$ changes direction (Fig. 2 b) and is now stationary in frame $S^{\prime}$, where the arm $\mathrm{AB}$ moves with relative velocity $v_{x^{\prime}}^{\prime}=-v$. Therefore, the rate of time of a clock is now the same as that of any clock co-moving with $S^{\prime}$, such as the clock $\mathrm{O}^{\mathrm{T}}$, facing point $\mathrm{A}$ at the moment of changing direction. At the turning point $\mathrm{A}$, clock $\mathrm{O}^{\mathrm{T}}$ needs to be accelerated in order to change direction. The corresponding acceleration $a$, which could cause a change in the rate of time as measured by clock $\mathrm{O}^{\mathrm{T}}$, may be assumed to depend on $v$ and the short path length $r$ along which it is acting. The time change $\tau=\tau[a(v, r)]$, due to the acceleration $a$ and corresponding to the time delay experienced by $\mathrm{O}^{\mathrm{T}}$, does not depend on $L=\mathrm{AB}$ and, if $L$ is large enough, $\tau$ becomes negligible - as assumed here - with respect to any time delay, such as $T$ (given by (2)), proportional to $L$.

For the circular case of Fig. 1, as measured by an observer co-moving with the measuring apparatus (or clock), the "ground" length of the closed path covered by the signal is $2 \pi R$. With the initial conditions chosen for the set-up of Fig. 1 (bottom) and Fig. 2, the observer co-moving with $\mathrm{O}^{\mathrm{T}}$ evaluates the path ground length covered by the signal to be $2 L$. In fact, with reference to Fig. 2 a) where $\mathrm{O}^{\mathrm{T}}$ is at $\mathrm{A}$, the signal has covered the arm $\mathrm{AB}=L$ from $\mathrm{O}^{\mathrm{T}}$ to $\mathrm{B}$. Since clock $\mathrm{O}^{\mathrm{T}}$ starts co-moving with frame $S^{\prime}$ at $\mathrm{A}$, we have $\mathrm{O}^{\mathrm{T}} \equiv \mathrm{O}^{\prime}$ and, after reflection at $\mathrm{B}$, the signal is expected to cover the remaining ground length $L$ from $\mathrm{B}$ to $\mathrm{O}^{\mathrm{T}} \equiv \mathrm{O}^{* \prime}$ at $\mathrm{A}$.

We may choose to apply Einstein or absolute synchronization in $S^{\prime}$. Yet, the physical reality of $S^{\prime \prime}$ cannot be altered by the choice of synchronization applied in $S^{\prime}$. We denote by $T=T_{A B}+\Delta T$ the round trip time displayed by clock $\mathrm{O}^{\mathrm{T}}$ when finally reached by the signal, being $\Delta T=\Delta t^{\prime}=L^{\prime} / C^{\prime}$ the time variation measured by $\mathrm{O}^{\mathrm{T}} \equiv \mathrm{O}^{\prime}$ for the propagation along the return path $L^{\prime}$, with $C^{\prime}$ representing the ground local speed in $S^{\prime}$. In line with SR (with either absolute or Einstein synchronization), the theoretical, or experimental, value of $T$ is $T=(T)_{\exp }=2 L /(c+v)$, as given by $t_{+}$in (2). Since, according to (Spavieri, Rodriguez \& Sanchez, 2018), synchronization is not arbitrary, the correct "natural" synchronization in $S^{\prime}$ must be the one consistent with the readings of clock $\mathrm{O}^{\mathrm{T}} \equiv \mathrm{O}^{\prime}$. Then, if the return path has length $L^{\prime}=L$, the round trip time is given by,

$$
T=T_{A B}+\Delta T=\frac{L}{c}+\frac{L^{\prime}}{C^{\prime}}=\frac{L}{c}+\frac{L}{C^{\prime}}=\frac{2 L}{c+v},
$$


a)

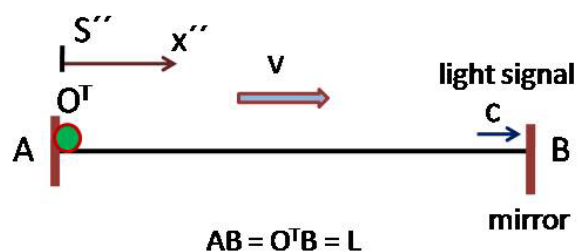

b)

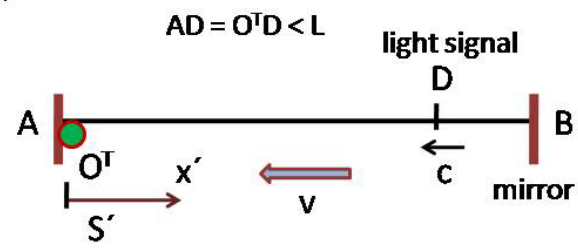

Figure 2: a) In frame $S^{\prime \prime}$, co-moving with the upper part of the belt where clock $\mathrm{O}^{\mathrm{T}}$ is stationary, the arm $\mathrm{AB}$ is moving with velocity $v$ in the $x^{\prime \prime}$ direction. At $t^{\prime \prime}=0$, simultaneously in $S^{\prime \prime}$, the end point A coincides with $\mathrm{O}^{\mathrm{T}}$ at the origin $\mathrm{O}^{\prime \prime}$, while the light signal has reached point $\mathrm{B}$ at the distance $x_{B}^{\prime \prime}=L$. The time of flight of the signal from $\mathrm{O}^{\mathrm{T}}$ to $\mathrm{B}$ is measured by clock $\mathrm{O}^{\mathrm{T}}$ as the time interval $\left.T_{A B}=L / c . b\right)$ After changing direction at $\mathrm{A}$, clock $\mathrm{O}^{\mathrm{T}}$ is stationary in frame $S^{\prime}$ co-moving with the lower part of the belt. Relatively to $S^{\prime}$, the arm $\mathrm{AB}$ is moving with velocity $-v$ in the $x^{\prime}$ direction and frame $S^{\prime \prime}$ with velocity $\simeq-2 v$. Due to non-conservation of simultaneity between $S^{\prime \prime}$ and $S^{\prime}$, when in frame $S^{\prime}$ clock $\mathrm{O}^{\mathrm{T}}$ and point $\mathrm{A}$ coincide, the light signal has already moved at $\mathrm{D}$ after being reflected at $\mathrm{B}$.

and from (8) we obtain the superluminal speed,

$$
C^{\prime}=\frac{L}{\Delta T}=\frac{L}{\Delta t^{\prime}} \simeq c+2 v,
$$

as foreseen by the AT, but in conflict with Einstein's second postulate.

\subsection{Propagation Time Measured by $O^{T}$ While Stationary in Frame $S^{\prime}$ According to Standard SR}

Result (9) has been obtained from considerations that reflect the reality of an observer of frame $S^{\prime \prime}$, where the signal reaches $\mathrm{B}$ at the same time when $\mathrm{A}$ reaches $\mathrm{O}^{\mathrm{T}}$. Let us see now how standard SR interprets result (8). For the purpose of simplifying calculations, we may assume that the origin $\mathrm{O}^{\prime \prime}$ of $S^{\prime \prime}$ coincides with the origin $\mathrm{O}^{\prime}$ of $S^{\prime}$ at $t^{\prime \prime}=t^{\prime}=0$ when clock $\mathrm{O}^{\mathrm{T}} \equiv \mathrm{O}^{\prime \prime}$ is at point $\mathrm{A}$ and, after changing direction, starts co-moving with frame $S^{\prime}$. Of course, clock $\mathrm{O}^{\mathrm{T}}$ has already accumulated the time $T_{A B}=L / C$ when is at A. By imposing Einstein synchronization to clocks in $S^{\prime}$, in order that the measured one-way light speed be $c$, relative simultaneity is introduced and the two events simultaneous in $S$ ", "light signal at $\mathrm{B}$ " and "clock $\mathrm{O}^{\mathrm{T}}$ at $\mathrm{A}$ ", are no longer simultaneous in $S^{\prime}$. In fact, the time transformation $t^{\prime}=t^{\prime \prime}-w x^{\prime \prime} / c^{2}$ gives $t_{B}^{\prime}=0-2 v L / c^{2}$ for the first event (at $x_{B}^{\prime \prime}=L, t_{B}^{\prime \prime}=0$ ) which precedes the second, $t_{A}^{\prime}=0-0\left(\right.$ at $x_{A}^{\prime \prime}=x_{O^{\prime \prime}}^{\prime \prime}=0, t_{A}^{\prime \prime}=0$ ) by the time interval,

$$
\delta t^{\prime}=t_{A}^{\prime}-t_{B}^{\prime}=2 L \frac{v}{c^{2}} .
$$

Thus, by means of non-conservation of simultaneity, standard SR changes scenario and we are transported from the physical reality of $S^{\prime \prime}$ to that of frame $S^{\prime}$, reflected respectively in Fig. 2 a) and 2 b): In the time interval $\delta t^{\prime}$ the signal has jumped by $\delta t^{\prime} c=2 L v / c$ past point $\mathrm{B}$ toward clock $\mathrm{O}^{\mathrm{T}} \equiv \mathrm{O}^{\prime}$. Hence, when for $S^{\prime}$ clock $\mathrm{O}^{\mathrm{T}} \equiv \mathrm{O}^{\prime}$ is at $\mathrm{A}$, the signal has already reached point $\mathrm{D}$ after being reflected at $\mathrm{B}$. It should be pointed out that the time interval (10), brought in as effect of nonconservation of simultaneity, corresponds to the "unphysical" time gap introduced by Kassner in the context of the Sagnac effect, a time discontinuity objected by Gift (2015) and Selleri (1997). With the now shorter light path DA of length $\ell^{\prime}=L^{\prime}=L-\delta t^{\prime} c=L(1-2 v / c)$ and with the ground speed $c=\ell^{\prime} / \Delta t_{E s}^{\prime}=\ell^{\prime} / \Delta T$ after Einstein synchronization, the LT foresee,

$$
(T)_{E s}=T_{A B}+\Delta T=\frac{A B}{c}+\frac{D A}{c}=\frac{L}{c}+\frac{\ell^{\prime}}{c}=2 \frac{L}{c}\left(1-\frac{v}{c}\right) \simeq \frac{2 L}{c+v},
$$


in agreement with (8). The last term in (11) is obtained by means of the power expansion $1 /(c+v)=1 /[c(1+v / c)] \simeq$ $(1 / c)(1-v / c)$ valid to the first order in $v / c$.

\subsection{Superluminal Local Speeds and the Missing Path Section}

In (8) and (11), the propagation time $T$ observed by means of the single clock $\mathrm{O}^{\mathrm{T}}$ can be interpreted, respectively, as due to:

a) Signal covering the closed ground path of length $2 L$ at the superluminal average local speed $c+v$, or

b) Signal covering the ground path of length $2 L(1-v / c)$ at the local speed $c$.

The correct interpretation must take into account internal consistency and reflect the evidence of the observer co-moving with clock $\mathrm{O}^{\mathrm{T}}$. Being $C=c$ the local light speed in frame $S^{\prime \prime}$, it is unquestionable that, at $T_{A B}=L / c$, the signal has covered the distance $\mathrm{AB}=L$ only, excluding the section $\mathrm{BD}=\delta t^{\prime} c$. Furthermore, if the return path is $L^{\prime}=L$, relatively to $\mathrm{O}^{\mathrm{T}} \equiv \mathrm{O}^{\prime}$ at $\mathrm{A}$, the return local speed is $C^{\prime} \simeq c+2 v$, while the average speed is $\simeq c+v$ over the round trip path $2 L$. Then, results (8) and (9), coherent with interpretation $a$ ), favor absolute synchronization.

Visibly, standard SR seems to imply interpretation $b$ ) instead. In this case, however, we are faced with an inconsistency by adopting Einstein synchronization in $S^{\prime}$. In fact, relying on the evidence that, for the observer co-moving with $\mathrm{O}^{\mathrm{T}}$, $t_{A B}^{*}=A B / c=L / c$ refers to the propagation along $\mathrm{AB}$, and considering that the term $\Delta t_{E s}^{\prime}=\ell^{\prime} / c$ in $(11)$ refers merely to the propagation along $\mathrm{DA}=\ell^{\prime}$, the path section $\mathrm{BD}=\delta t^{\prime} c$ (and the corresponding time contribution) is missing in (11), being removed by the introduction of non-conservation of simultaneity. Indeed, if we restore the missing path section, Eq. (11) becomes,

$$
(T)^{E s}=\frac{L}{c}+\frac{\ell^{\prime}}{c}+\frac{\delta t^{\prime} c}{c}=\frac{2 L}{c},
$$

in line with the second postulate but in disagreement with the expected value $T=(T)_{\exp }$.

We may try to justify the absence of the path section $\mathrm{BD}=\delta t^{\prime} c$ by including $\delta t^{\prime}$ in either $T_{A B}$ or $\Delta t_{E s}^{\prime}$ in (11). However, if Einstein synchronization is applied to both $S^{\prime \prime}$ and $S^{\prime}$, we face an irreconcilable discrepancy between the reality of frame $S^{\prime \prime}$ and that of $S^{\prime}$ : According to $S^{\prime \prime}$, the path section $\mathrm{BD}=\delta t^{\prime} c$ has been covered by the signal after point $\mathrm{A}$ and $\mathrm{O}^{\mathrm{T}}$ meet and, thus, $\delta t^{\prime}$ is not included in the time interval $T_{A B}$. According to $S^{\prime}$, instead, it has been covered before and, thus, $\delta t^{\prime}$ is not included in the time interval $\Delta t_{E s}^{\prime}$. But let us suppose, for example, that $S^{\prime}$ is right and, then, according to $S^{\prime}$, we have to include $\delta t^{\prime}$ in $T_{A B}$. In this case, we force the observer $\mathrm{O}^{\mathrm{T}}$ to acknowledge (contrary to observation) that the signal has covered the ground distance $\mathrm{AB}=L$ plus the section $\delta t^{\prime} c$ in the interval $T_{A B}$, implying that the local speed along this path is $\left(L+\delta t^{\prime} c\right) / T_{A B}=c+2 v$, i.e., superluminal. The point is that the contention of $S^{\prime}$, that at $T_{A B}$ the signal has covered also the section $\mathrm{BD}=\delta t^{\prime} c$, is questionable because based on readings of clocks of $S^{\prime}$ not co-moving with $\mathrm{O}^{\mathrm{T}}$ and because $\mathrm{BD}$ is not a ground path for $\mathrm{O}^{\mathrm{T}}$, as required by (6). Similar conclusions hold if $\delta t^{\prime}$ is included in $\Delta t_{E s}^{\prime}$. It follows that, since the signal has to cover also the missing section $\mathrm{BD}$, and given that the average light speed $c+v$ is superluminal, if the local speed is $c$ in one part of the path, in the remaining part the local speed must be superluminal and vice versa.

\section{Conclusions}

In the non-relativistic approximation, the spin-orbit mechanism can be interpreted in the frame of the nucleus by taking into account either the hidden momentum of the orbiting electron or the precession of the electron spin derived by Thomas. So far, we do not know of dedicated tests confirming that the Thomas precession is observable. Thus, if the existence of the hidden momentum is confirmed by observation, the interpretation of the spin-orbit mechanism in terms of absolute simultaneity is favored.

As is well-known (Kassner, 2012; Weber, 1997; Anandan, 1981; Gift, 2015), Einstein synchronization fails when performed along a closed path. The analysis of our thought experiment - equivalent to the linear Sagnac effect - confirms that the local one-way speed of the signal cannot be $c$ along the closed ground path $2 L$, in line with Selleri's paradox and in conflict with Einstein's second postulate. As long as the light signals form closed trajectories, applying Einstein synchronization to the closed paths, or separately to both frames $S^{\prime \prime}$ and $S^{\prime}$ in our thought experiment, leads to the same failure. By means of Einstein synchronization applied to the inertial frame $S^{\prime \prime}$, we may verify the simultaneity of events within frame $S^{\prime \prime}$. Similar conclusions can be drawn within frame $S^{\prime}$. However, our thought experiment shows that the two simultaneities, simultaneity within $S^{\prime \prime}$ and simultaneity within $S^{\prime}$, are incompatible with relative simultaneity and the related Einstein's second postulate because it introduces missing light paths and superluminal light speeds. Since the interpretation of the Sagnac effect is consistent with the "natural" absolute synchronization, if clock synchronization were arbitrary, the mentioned inconsistencies related to Einstein synchronization could be waived by switching synchronization. Nevertheless, as shown in Ref. (Spavieri, Rodriguez \& Sanchez, 2018), the underlying realities of Einstein and absolute synchronization are physically and conceptually dissimilar and, thus, the two synchronizations, as well as relative and absolute simultaneity, cannot be arbitrarily interchanged. If we assume as valid the principle that a physical 
theory must describe reality without contradicting its own postulates, then, to interpret the linear Sagnac effect coherently, SR requires absolute synchronization. In this case, if it exists, the preferred frame can be identified by performing experiments such as those indicated in Ref. (Spavieri, Rodriguez \& Sanchez, 2018). As a possible candidate for preferred frame theories based on absolute synchronization, some authors (Selleri, 1997; Spavieri, 2012; Gift, 2015; Spavieri, Rodriguez \& Sanchez, 2019; Cavalleri, Galgani, Spavieri \& Spinelli, 1976) suggest the Earth Centered Inertial (ECI) or the Sun Centered Inertial (SCI) frame, which could fit into the pre-relativistic scenario of a Stokes-Planck (Stokes, 1845) ether theory capable of interpreting all optical experiments (Cavalleri, Galgani, Spavieri \& Spinelli, 1976).

In closing, we note that beyond paradoxes or inconsistencies with Einstein synchronization, all these discussions show that the formulation of SR with absolute synchronization is an interesting approach, given that all the known experiments supporting SR can be interpreted with either absolute or relative synchronization (Mansouri \& Sexl, 1977). Hence, one possible course of action when employing the Lorentz transformations might consider exploring how the routinely used models and theoretical applications change when transformations based on absolute simultaneity are used instead. This may lead to interesting new insights that might aid to discriminate absolute from relative simultaneity, as investigated for instance in Ref. (Spavieri, Rodriguez \& Sanchez, 2018), thus helping to advance our understanding of relativistic theories.

\section{Appedix}

Among detractors of the Thomas mechanism, we mention Fisher, who states (Fisher, 1972) that 'Thomas's original paper...got the correct answer by an incorrect physical argument. This wrong argument persists to this day, so let us hasten to correct it.' It is also worth mentioning that the BMT equation (Bargmann, Michel \& Telegdi, 1959) for the electron spin, was derived in 1959, while the correct expression for the force acting on the electron magnetic dipole has been found (Aharonov, Pearle \& Vaidman) later, in 1988. Thus, the BMT equation does not take into account the extra force and torque on the electron due to the hidden momentum.

The Referees of the articles (Spavieri \& Mansuripur, 2015) and (Spavieri, 2016) pointed out that the difficulty with all the Thomas precession derivations is to obtain it directly working in the frame $S$ of the nucleus, and not in the frame of the electron, as generally done. At this point, we consider convenient to re-examine the well-known derivations of the Thomas precession by Jackson (1999), and Page and Adams (1965). The idea is to emphasize how both derivations link the Thomas precession to the effect of non-conservation of simultaneity, and point out possible drawbacks.

\subsection{Jackson's Derivation}

Let us consider a particle (e.g., an electron) moving on a plane in circular motion with constant velocity $\mathbf{v}$ with respect to the laboratory frame $S$. Let $S^{\prime}$ be an inertial frame instantaneously co-moving with the particle with velocity components $v_{x}=v \cos \varphi$ and $v_{y}=v \sin \varphi$. The Lorentz transformations between the lab frame $S$ and the rest frame $S^{\prime}$ are,

$$
\mathbf{x}^{\prime}=\mathbf{x}+\frac{\gamma-1}{v^{2}}(\mathbf{v} \cdot \mathbf{x}) \mathbf{v}-\gamma \mathbf{v} t ; \quad t^{\prime}=\gamma\left(t-\mathbf{v} \cdot \mathbf{x} / c^{2}\right) .
$$

At $\varphi=0$, the particle rest frame $S^{\prime}$ will be moving with velocity $v_{x}=v$ with respect to $S$. As shown by Jackson (1999), Thomas introduces also the rest frame $S^{\prime \prime}$ of the particle when, in its circular motion, reaches the position $\psi=\varphi+\varphi_{0}$. The velocity components of $S^{\prime \prime}$ are $v_{x}^{\prime}=v \cos \psi$ and $v_{y}^{\prime}=v \sin \psi$ and, for $\varphi=0$ and $\varphi_{0}$ small, $v_{x}^{\prime}=v$ and $v_{y}^{\prime}=\varphi_{0} v$. Using (13) to express the $x^{\prime \prime}$ coordinate as function of $x^{\prime}$, Jackson finds ( $\varphi=0$ and $\varphi_{0}$ small)

$$
\begin{aligned}
& x^{\prime \prime}=x^{\prime}+(\gamma-1) \varphi_{0} y^{\prime} \\
& y^{\prime \prime}=-(\gamma-1) \varphi_{0} x^{\prime}+y^{\prime}-\gamma \varphi_{0} v t^{\prime} .
\end{aligned}
$$

Eq. (14) represents a boost from $S^{\prime}$ to $S^{\prime \prime}$ plus a relative rotation $\Omega=(\gamma-1) \varphi_{0}$ about the $z^{\prime}$ axis. At the time $t^{\prime}=0$, simultaneously in frame $S^{\prime}$, the relative rotation between $S^{\prime \prime}$ and $S^{\prime}$ is apparent because the diagonal coefficient $(\gamma-1)$ in (14) changes sign. Thus, according to Jackson, under specific assumptions, $-\Omega$ can be interpreted, as done by Thomas, as the rotation acquired by the spin fixed to the physical axes of the particle when it "jumps" from frame $S^{\prime}$ to $S^{\prime \prime}$. The resulting Thomas precession frequency is

$$
\omega_{T}=-\delta \mathbf{\Omega} / \delta t=\gamma^{2}(\gamma+1)^{-1} \mathbf{a} \times \mathbf{v} / c^{2},
$$

where the angular frequency $\omega_{T}$ is derived with the additional assumption $\varphi_{0}=\omega t$, with $\omega$ the angular frequency and $\mathbf{a}=\omega^{2} \mathbf{r}$ the particle acceleration. For the purposes of our paper, we need not to consider the hypotheses made in deriving $\omega_{T}$, but we may focus on the rotation $\Omega$ reflected by Eq. (14), prerequisite to derive the Thomas precession frequency $\omega_{T}$.

The transformations from $S$ to $S^{\prime}$ and from $S$ to $S^{\prime \prime}$ are pure boosts (Lorentz transformation (13) without rotation) and, thus, we expect that, as measured by an observer at rest in the lab frame $S$, there is no relative rotation between frame $S^{\prime \prime}$ 
and $S^{\prime}$. The absence of rotation can be shown by deriving the transformations (14) in function of the time $t$ and with $\varphi_{0}$ small, as seen by an observer in the lab frame $S$ and simultaneously in $S$, rather than simultaneously in frame $S^{\prime}$ at the time $t^{\prime}$. Since $t=\gamma\left(t^{\prime}+v x^{\prime} / c^{2}\right)$, we have $\gamma t^{\prime}=t-\gamma v x^{\prime} / c^{2}$, which can be substituted in (14). Factorizing $\varphi_{0} x^{\prime}$ and with $\gamma\left(1-v^{2} / c^{2}\right)=\gamma^{-1}$, the coordinate transformations between $S^{\prime}$ and $S^{\prime \prime}$ as function of $t$ are given by,

$$
\begin{aligned}
& x^{\prime \prime}=x^{\prime}+(\gamma-1) \varphi_{0} y^{\prime} \\
& y^{\prime \prime}=(\gamma-1) \gamma^{-1} \varphi_{0} x^{\prime}+y^{\prime}-\varphi_{0} v t .
\end{aligned}
$$

The diagonal coefficients of (16) have the same sign and, therefore, transformations (16) prove that, as expected, there is no relative rotation between frame $S^{\prime \prime}$ and $S^{\prime}$ at $t=0$, simultaneously in $S$. In conclusion, as the electron advances in its circular trajectory around the nucleus, there is no spin rotation for an observer stationary in the laboratory (or nucleus) frame $S$. In fact, in (14) and (16), $\varphi_{0}$ is more properly expressed as $\varphi_{0}=\omega t_{0}=$ const. $\neq 0$ ( $t_{0}$ small). Thus, the "rotation" $\Omega=(\gamma-1) \varphi_{0}$ corresponding to transformations $(14)$ is constant $(d \Omega / d t=0)$. In this case, transformations $(14)$ and (16) can be interpreted as reflecting the different relative axes distortion due to the Lorentz-Fitzgerald contraction and non-conservation of simultaneity between the two frames $S^{\prime \prime}$ and $S^{\prime}$, as perceived simultaneously by an observer of $S^{\prime}$ or simultaneously by an observer of $S$, respectively. On account of (16), showing that there is no rotation for $S$, in no way $\Omega$ can be interpreted as a spin rotation, or precession, that is being accumulated and is increasing with time in the nucleus frame $S$.

\subsection{Page and Adams' Derivation}

In the derivations of Thomas and Jackson, the axes distortions, or "rotation", of Eq. (14) is assumed "ad hoc" to lead to the continuos spin precession at the Thomas frequency $\omega_{T}$ as perceived by the observer of frame $S^{\prime}$ co-moving with the electron. However, the challenge is to derive the Thomas precession in the lab frame $S$, where the nucleus is at rest and the spin-orbit energy splitting is observed. This task has been attempted by Page and Adams (1965) by introducing an arrow $O P$ representing the spin vector $\mathbf{p}$, where the terminus $O$ is fixed to the electron moving with velocity $\mathbf{v}$ relatively to the laboratory frame $S$, and the terminus $P$ has velocity $\mathbf{V}$ relatively to the frame $S$ where the nucleus is at rest. Page and Adams use a non-standard original approach to derive the displacement of $P$, relatively to $O$, due to the effect of non-conservation of simultaneity between $S^{\prime}$ and $S$. After a series of assumptions, they obtain the following equation for the spin vector $\mathbf{p}$,

$$
\mathbf{p}=\mathbf{p}^{\prime}+(\gamma-1) \mathbf{p}^{\prime} \cdot \hat{\mathbf{v}} \hat{\mathbf{v}}-\gamma \frac{\mathbf{p}^{\prime} \cdot \mathbf{v}}{c^{2}} \mathbf{V}
$$

In the subsequent step by Page and Adams, the main assumption is, $d \mathbf{p}^{\prime} / d t^{\prime}=0$ always in the co-moving frame $S^{\prime}$. Therefore, we may set $\mathbf{p}^{\prime}=\overline{\mathbf{p}^{\prime}}=$ const in (17). Then, we get,

$$
\mathbf{p}=\overline{\mathbf{p}^{\prime}}+(\gamma-1) \gamma \overline{\mathbf{p}^{\prime}} \cdot \hat{\mathbf{v}} \hat{\mathbf{v}}-\gamma^{2} \frac{\overline{\mathbf{p}} \cdot \mathbf{v}}{c^{2}} \mathbf{V}=\overline{\mathbf{p}}-\gamma \frac{\overline{\mathbf{p}^{\prime}} \cdot \mathbf{v}}{c^{2}} \mathbf{V}
$$

In (18) $\overline{\mathbf{p}}$ and $\overline{\mathbf{p}^{\prime}}$ are constant and, therefore, in frame $S$ we may interpret $\mathbf{p}=\mathbf{p}(t)$ as given mainly by the fixed, nonrotating constant vector $\overline{\mathbf{p}}$ plus other second order terms that represent small oscillations of the spin about $\overline{\mathbf{p}}$, in line with what discussed above about result (16).

Furthermore, after deriving (17), Page and Adams finds the following equation of motion for the spin vector $\mathbf{p}$,

$$
\frac{d \mathbf{p}}{d t}=-\gamma^{2} \frac{\mathbf{p} \cdot \mathbf{v}}{c^{2}} \frac{d \mathbf{V}}{d t}=-\gamma^{2} \frac{\mathbf{p} \cdot \mathbf{v}}{c^{2}} \mathbf{A}
$$

After more assumptions, including motion in the plane of the closed trajectory and $\mathbf{v}$, Page and Adams obtain from (19) the equation,

$$
\frac{d \mathbf{p}}{d t}=\omega_{T} \times \mathbf{p}
$$

where $\omega_{T}$ denotes the Thomas angular precession, derived by them as,

$$
\omega_{T}=\frac{\mathbf{p}}{P^{2}} \times \frac{d \mathbf{p}}{d t}=-\gamma^{2} c^{-2}(\widehat{\mathbf{p}} \cdot \mathbf{v}) \widehat{\mathbf{p}} \times \mathbf{A},
$$

where $\widehat{\mathbf{p}}=\mathbf{p} / p$.

For the circular motion of the electron orbiting the nucleus, the Thomas angular precession is constant, so that in (21) $\omega_{T}$ must be constant, in this case. The problem with Page and Adams' derivation, once accepting the various assumptions made, is that $\omega_{T}$ in (21) is not constant, being proportional to $\widehat{\mathbf{p}}=\widehat{\mathbf{p}}(t)$ and to $\mathbf{A}=d \mathbf{V} / d t$, the acceleration of the terminus $P$ 
of the rotating spin arrow. Thus, Page and Adams' result for the precession of $\mathbf{p}=\mathbf{p}(t)$, derived in frame $S$, is questionable because it appears to have no relation with the precession mechanism proposed by Thomas for the interpretation of the spin-orbit energy splitting.

\section{Acknowledgments}

This work was supported by the following institutions: CDCHTA of the Universidad de Los Andes, Mérida, Venezuela; Ecological World For Life GmbH, D-15537 Erkner, Germany; Istituto Nazionale di Fisica Nucleare (INFN), Università di Udine; International Centre for Theoretical Physics, Trieste, Italy.

\section{References}

Aharonov, Y., Pearle, P., \& Vaidman, L. (1988). Comment on "Proposed Aharonov-Casher effect: Another example of an Aharonov-Bohm effect arising from a classical lag", Phys. Rev. A, 37, 4052; Vaidman, L. (1990). Torque and force on a magnetic dipole, Am. J. Phys., 58, 978-983; Hnizdo, V. (2012), Comment on 'Electromagnetic force on a moving dipole', Eur. J. Phys., 33, L3.

Anandan, J. (1981). Sagnac effect in relativistic and nonrelativistic physics, Phys. Rev., D, 24, 338-346. https://doi.org/10.1103/PhysRevD.24.338

Bargmann, V., Michel, L., \& Telegdi, V. L. (1959). Precession of the Polarization of Particles Moving in a Homogeneous Electromagnetic Field, Phys. Rev. Lett. 2, 435. The derivation of the BMT equation, however, does not take into account the electron hidden momentum. https://doi.org/10.1103/PhysRevLett.2.435

Bell, J. S. (1988). Speakable and Unspeakable in Quantum Mechanics (Cambridge Univ. Press, Cambridge).

Cavalleri, G., Galgani, L., Spavieri, G., \& Spinelli, G. (1976). Classical optics experiments and the ether, Scientia, 111(9-10-11-12), 675-680.

de Abreu, R., \& Guerra, V. (2005). The conceptualization of time and the constancy of the speed of light, Eur. J. Phys., 26, 117-123 ; (2006). On the Consistency between the Assumption of a Special System of Reference and Special Relativity, Found. Phys., 36, 1826-1845; (2008). The principle of relativity and the indeterminacy of special relativity, Eur. J. Phys., 29, 33-52; Selleri, F. (2005). The Inertial Transformations and the Relativity Principle, Found. Phys. Lett., 18, 325-339.

Discussions on clock synchronization may be found in: Anderson, R., Vetharaniam, I., \& Stedman, G. E. (1998). Conventionality of synchronization, gauge dependence and test theories of relativity, Phys. Rep. 295, 93-180; Ohanian, H. C. (2004). The role of dynamics in the synchronization problem, Am. J. Phys., 72, 141-148; Spavieri, G. (2012). On measuring the one-way speed of light, Eur. Phys. J. D, 66, 76; Spavieri, G., \& et al. (2012). Can the one-way speed of light be used for detection of violations of the relativity principle? Phys. Lett. A, 376 795-797.

Fisher, G. P. (1972). The Thomas precession. Am. J. Phys., 40, 1772-81. https://doi.org/10.1119/1.1987061

Gift, S. J. G. (2015). On the Selleri Transformations: Analysis of Recent Attempts by Kassner to Resolve Selleri's Paradox, Applied Physics Research, 7(2). https://doi.org/10.5539/apr.v7n2p112

Jackson, J. D. (1999). Classical Electrodynamics (3rd ed. New York: Wiley) sections 11-8 and 11-11. https://doi.org/10.1119/1.19136

Kholmetskii, A. L., Missevitch, O. V., \& Yarman, T. (2010). On the classical analysis of spin-orbit coupling in hydrogenlike atoms. Am. J.Phys. 78, 428. https://doi.org/10.1119/1.3277052

Khorrami, M. (2013). Comment on Trouble with the Lorentz Law of Force: Incompatibility with Special Relativity and Momentum Conservation. Phys. Rev. Lett. 110, 089404; Saldanha, P. (2013). Comment on Trouble with the Lorentz Law of Force: Incompatibility with Special Relativity and Momentum Conservation. Phys. Rev. Lett. 110, 089403

Kemp, B. (2015). Macroscopic Theory of Optical Momentum. Prog. Opt. 60, 437-488.

Kassner, K. (2012). Ways to resolve Selleri's paradox. Am. J. Phys. 80, 1061.

Mansouri, R., \& Sexl, R. U. (1977). A test theory of special relativity. Gen. Rel. Grav., 8, 497, 515, 809.

On the Sagnac effect see: Spavieri, G., \& et al. (2019). to be submitted for publication. See also: Klauber, R. D. (2003). Derivation of the General Case Sagnac Result Using Non-Time-Orthogonal Analysis. Found. Phys. Lett., 16(5), 447; Field, J. H. (2017). The Sagnac effect and transformations of relative velocities between inertial frames. Fund. J. Modern Phys., 10(1), 1-30.

Page, L., \& Adams, N. I. jr. (1965) Electrodynamics (Dover Publications, Inc., New York). Sec. 44. For other derivations 
of the Thomas precession see: Malykin, G. B. (2006). Thomas precession: correct and incorrect solutions. Phys. Usp., 49(8), 83; Rindler, W. (2006) [2001]. "Chapter 9". Relativity Special, General and Cosmological, (2nd ed.). Dallas: Oxford University Press. ISBN 978-0-19-856732-5; Sexl, R. U., \& Urbantke, H. K. (2001) [1992]. Relativity, Groups Particles. Special Relativity and Relativistic Symmetry in Field and Particle Physics. Springer. 38-43. ISBN 978-3211834435

Sagnac, G. (1913). L'éther lumineux démotré par l'effet du vent relatif d'éther dans un intertféromètre en rotation uniforme. C. R. Acad. Sci., 157, 708-710.

Selleri, F. (1997). Non Invariant One Way Speed of Light and Locally Equivalent Reference Frames. Found. Phys. Lett., 10, 73-83; (2004b). Recovering the Lorentz Ether. Apeiron, 11, 246-281.

See, for example, Spavieri, G. (2012). On measuring the one-way speed of light. Eur. Phys. J., D 66, 76; Spavieri, G. \& Gillies, G. T. (2016). Angular momentum and the electromagnetic top. Pramana - J. Phys., 87, 19 c Indian Academy of Sciences. https://doi.org/10.1007/s12043-016-1219-5.

Shockley, W., \& James, R. R. (1967). ”Try Simplest Cases” Discovery of "Hidden Momentum” Forces on "Magnetic Currents". Phys. Rev. Lett., 18, 876.

Spavieri, G., \& Mansuripur, M. (2015). Origin of the spin-orbit interaction. Phys. Scr., 90, 085501.

Spavieri, G. (2016). Role of the electromagnetic momentum in the spin-orbit interaction. Eur. Phys. J., D 70, 263. https://doi.org/10.1140/epjd/e2016-60575-1

Spavieri, G., Rodriguez, M., \& Sanchez, A. (2018). Thought experiment discriminating Special Relativity from Preferred Frame Theories. J. Phys. Commun., 2, 085009. https://doi.org/10.1088/2399-6528/aad5fa

Spavieri, G. (1998). Comment on Quantum Phase of a Moving Dipole. Phys. Rev. Lett., 81, 1533; (1999). Quantum effect for an electric dipole. Phys. Rev. A, 59, 3194; (2003). Momentum of electromagnetic fields, speed of light in moving media, and the photon mass. Phys. Lett. A, 310, 13.

Stokes, G. G. (1845). Phil. Mag. 27, 9, reprinted in Mathematical and Physical Papers (1880). Cambridge U. P. Cambridge, 1, 134 .

Thomas, L. H. (1926). The motion of the spinning electron. Nature (London) 117, 514; 1927, The kinematics of an electron with an axis. Phil. Mag., 3, 1-22.

Tangherlini, F. R. (1961). Galilean-Like Transformation Allowed by General Covariance and Consistent with Special Relativity. Nuovo Cimento Suppl., 20,1. The Tangherlini transformations are known also as the IST (Inertial Synchronized - Tangherlini) transformations (de Abreu \& Guerra, 2005), and the Selleri transformations (Selleri, 1997).

Weber, T. A. (1997). Measurements on a rotating frame in relativity, and the Wilson and Wilson experiment. Am. J. Phys., 65, 946-953. https://doi.org/10.1119/1.18696

Wang, R., Zhengb, Y., Yaob, A., \& Langley, D. (2003). Modified Sagnac experiment for measuring travel-time difference between counter-propagating light beams in a uniformly moving fiber. Phys. Lett. A, 312, 7-10. https://doi.org/10.1016/S0375-9601(03)00575-9

\section{Copyrights}

Copyright for this article is retained by the author(s), with first publication rights granted to the journal.

This is an open-access article distributed under the terms and conditions of the Creative Commons Attribution license (http://creativecommons.org/licenses/by/4.0/). 